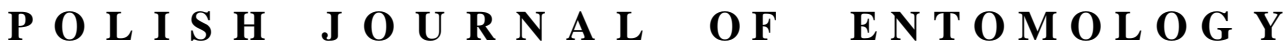

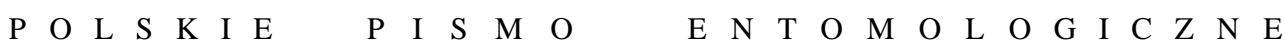

VOL. 86: 119-140

Lublin

30 June 2017

DOI: $10.1515 /$ pjen-2017-0009

\section{An annotated checklist of the aquatic Polyphaga (Coleoptera) of Egypt III. Families: Helophoridae, Georissidae, Hydrochidae, Spercheidae, Scirtidae, EImidae, Dryopidae, Limnichidae and Heteroceridae}

\author{
MOHAMED SALAH ${ }^{1,2 *}$ \\ ${ }^{1}$ Department of Zoology \& Entomology, Faculty of Science, Helwan University, \\ 11795 - Helwan, Cairo, Egypt \\ ${ }^{2}$ Department of Biology, Faculty of Applied Medical Sciences, Turabah Branch, \\ Taif University, P.O. Box 311, 21995 - Taif, Saudi Arabia
}

\begin{abstract}
An annotated list of the aquatic Polyphaga (Coleoptera: Helophoridae, Georissidae, Hydrochidae, Spercheidae, Scirtidae, Elmidae, Dryopidae, Limnichidae and Heteroceridae) recorded from Egypt is presented, based on data gathered from earlier literature. The checklist includes data on the type localities, references in which the species are described in detail, world and Egyptian distributions, and previous literature, for 21 valid species belonging to 13 genera: Helophorus (Helophoridae); Georissus (Georissidae); Hydrochus (Hydrochidae); Spercheus (Spercheidae); Contacyphon (Scirtidae); Oulimnius and Potamodytes (Elmidae); Dryops and Parahelichus (Dryopidae); Bothriophorus and Pelochares (Limnichidae); Augyles and Heterocerus (Heteroceridae). The present study provides a summary that can provide a basis for future progress in the knowledge of the Egyptian aquatic Coleoptera.
\end{abstract}

KEY WORDS: Aquatic beetles, Coleoptera, Polyphaga, Egypt.

\section{INTRODUCTION}

Hydrophiloidea, in the narrow sense, comprises the families Hydrophilidae, Helophoridae, Georissidae, Hydrochidae, Spercheidae and Epimetopidae. In a wider sense, it also includes Sphaeritidae, Synteliidae and Histeridae (ARCHANGELSKY et al. 2016). Five of these

\footnotetext{
* Corresponding author: msalahcoleo@gmail.com
} 
families have been reported in Egypt: Hydrophilidae, Helophoridae, Georissidae, Hydrochidae and Spercheidae. The monogeneric Helophoridae (grooved water scavenger beetles) has 192 species of the genus Helophorus FABRICIUS, 1775. Adults of most species are considered truly aquatic, inhabiting shallow stagnant water bodies. A few species are strictly terrestrial, live under stones or are found in open grassland. All known larvae are terrestrial, most are predaceous, but a few are herbivorous (ANGUS 1992, JÄCH 1998, FIKÁČEK 2009, ARCHANGELSKY et al. 2016). Georissidae (minute mud-loving beetles) are a monogeneric family with 80 known species described from a single genus Georissus LATREILLE, 1809. Adults and larvae of this family are usually riparian, inhabiting the wet sandy and muddy margins of rivers and streams. Some members can also inhabit stagnant and temporary water bodies, and a few species inhabit leaf-litter in tropical forests. Adults are saprophagous, whereas larvae are predaceous (FIKÁČEK \& TRÁVNíČEK 2009, ARCHANGELSKY et al. 2016). Hydrochidae (elongated water scavenger beetles) is a rather small family, comprising only one genus Hydrochus LEACH, 1817 and 181 species worldwide. Adults and larvae are aquatic, living in association with aquatic plants on the banks of stagnant or slow flowing water habitats (JÄCH 1998, ARCHANGELSKY et al. 2016). Spercheidae (filter-feeding water scavenger beetles) is a rather small family, comprising only one genus Spercheus KUGELANN, 1798 and 18 species. Both larvae and adults are aquatic, inhabiting stagnant water. Little is known about the biology of this family (ARCHANGELSKY 2001, 2016). The family Hydrophilidae (water scavenger beetles), is by far the most diverse family of Hydrophiloidea and has been treated in another paper (SALAH \& RÉGIL in press).

Scirtoidea is a superfamily of beetles in the Polyphaga. There are four families in this small superfamily: Clambidae, Decliniidae, Scirtidae and Eucinetidae (LAWRENCE 2016a). Scirtidae (marsh beetles) is the only family of Scirtoidea reported from Egypt. Scirtid larvae are usually aquatic whereas adults are generally terrestrial (but adults of Hydrocyphon REDTENBACHER, 1858 are occasionally collected under water) (JÄCH 1998). The family includes more than 1600 currently recognized species and 60 genera worldwide, but many species remain undescribed (LAWRENCE 2016b).

The superfamily Byrrhoidea includes 12 families: Byrrhidae, Elmidae, Dryopidae, Lutrochidae, Limnichidae, Heteroceridae, Psephenidae, Cneoglossidae, Ptilodactylidae, Chelonariidae, Eulichadidae and Callirhipidae (BEUTEL 2016). Four of these families (Elmidae, Dryopidae, Limnichidae and Heteroceridae) have been reported from Egypt. Elmidae (riffle beetles) is a moderately large, cosmopolitan family with 151 genera and almost 1500 species. Adults and larvae are usually considered to be aquatic, living in lotic habitats, while a few species are encountered in lentic habitats (lakes or ponds) (JÄCH 1998, JäCH et al. 2016). Dryopidae (long-toed water beetles) are a small family, with approximately 300 species in 33 genera. Adults occur in aquatic, riparian or terrestrial 
habitats. Many species can be definitely termed "True Water Beetles" (JÄCH 1998). Most of the known larvae are reported to be terrestrial, occurring in moist soil, sand, among decaying plant tissues or under decaying wood (KoDADA et al. 2016). The family Limnichidae (minute marsh-loving beetles) has about 37 genera and 387 known species worldwide. Most of the species seem to be semiaquatic or riparian, found on the shores of freshwater habitats, often very close to the water's edge (HERNANDO \& RIBERA 2016a). Heteroceridae contains small beetles commonly known as variegated mud-loving beetles. This family includes about 300 species from five genera worldwide. Adults and larvae are typical shore beetles, which dig tunnels in wet sand and mud, preferably near freshwater (MASCAGNI 2014).

African beetles have received considerable attention from many researchers. However, descriptions, taxonomic notes and the distribution of most Egyptian species are scattered about the old literature, which is often accessible only with considerable difficulty. The aim of the present work is to complete a series of studies carried out by SALAH \& RÉGIL (2014a, 2014b, 2015, in press) and SALAH et al. (2014) by integrating all published sources and bringing together all the scattered literature on the families Helophoridae, Georissidae, Hydrochidae, Spercheidae, Scirtidae, Elmidae, Dryopidae, Limnichidae and Heteroceridae, in order to provide a summary that can serve as the basis for future progress in the knowledge of these families.

\section{Acknowledgements}

I would like to thank Dr Juan Antonio RÉGIL CUETO (Department of Biodiversity and Environmental Management, León University, Spain) for his generous and unfailing support of my research and for his valuable suggestions on revising the original manuscript.

\section{MATERIAL AND METHODS}

\section{Biogeography of Egypt}

Egypt is a transcontinental country spanning the north-east corner of Africa and the south-west corner of Asia by a land bridge formed by the Sinai Peninsula. With a surface area of about one million $\mathrm{km}^{2}$, the country lies at the centre of the largest and driest desert region in the world. Average temperatures range from 20 to $30^{\circ} \mathrm{C}$ during summer, and from 10 to $20^{\circ} \mathrm{C}$ in winter. The mean annual rainfall over most of the country is less than $10 \mathrm{~mm}$ (BAHA El DIN 2001). According to HOATH (2003), the lands of Egypt can be divided into six distinct areas: the Nile Valley and Delta, the Eastern Desert, the Western Desert, the Northern coastal strip, the Sinai Peninsula and Gebel Elba (Fig. 1). 


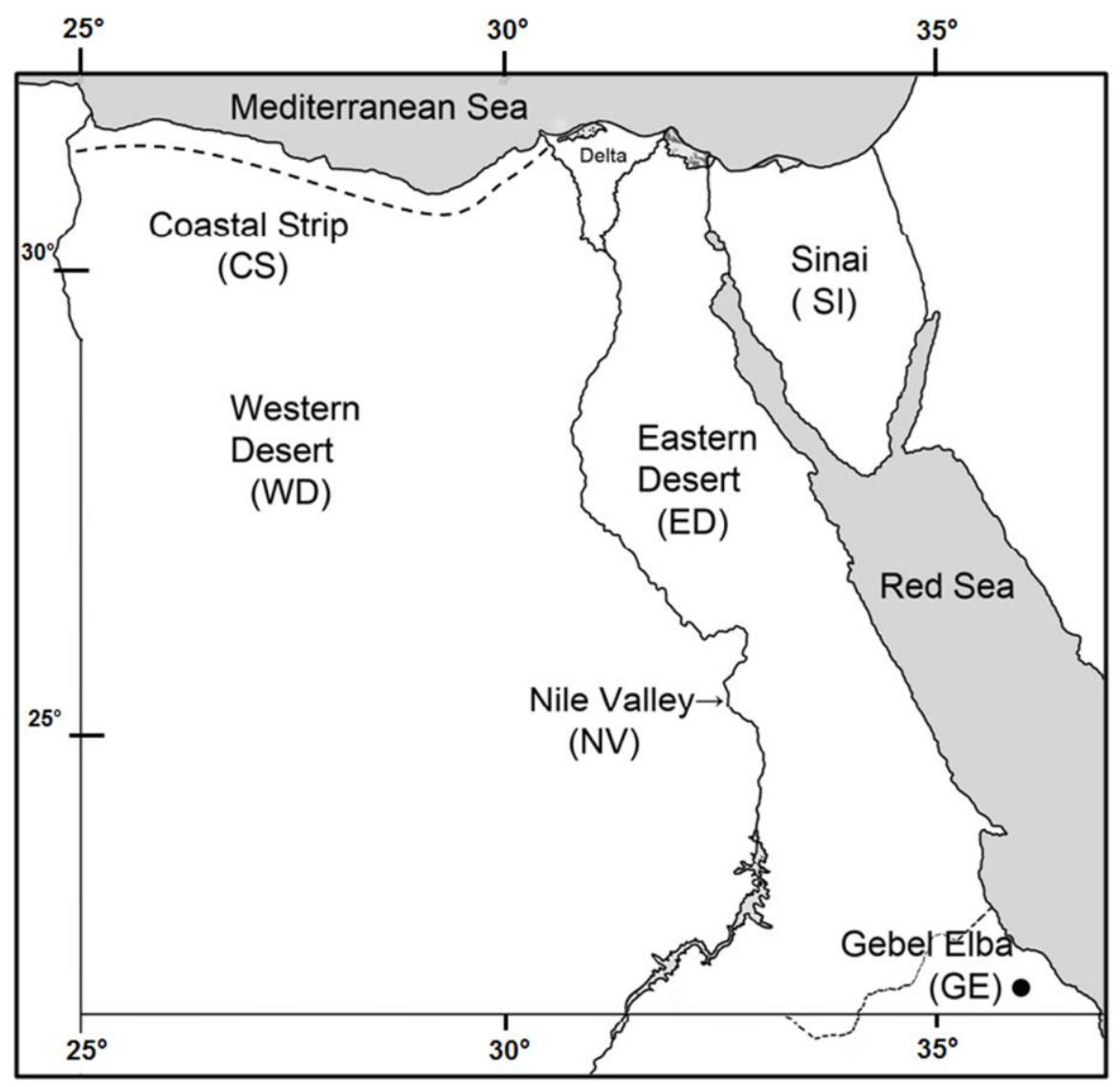

Fig. 1. Map of the Egyptian bioregions. (CS) Northern Coastal Strip; (ED) Eastern Desert; (GE) Gebel Elba; (NV) Nile Valley and Delta; (SI) Sinai Peninsula; (WD) Western Desert.

\section{Checklist arrangement}

Classification and the nomenclature of the target families largely follow FIKÁČEK et al. (2015) (Helophoridae), FIKÁČEK \& PRZEWOŹNY (2015) (Georissidae), HANSEN (1999) (Hydrochidae), FIKÁČEK (2015) (Spercheidae), KLAUSNITZER (2016a) (Scirtidae), JÄCH \& KODADA (2016) (Elmidae), KODADA \& JÄCH (2016) (Dryopidae), HERNANDO \& RIBERA (2016b) (Limnichidae) and MASCAGNI (2016) (Heteroceridae).

The present checklist includes all valid names of extant beetle taxa belonging to these 9 families, known to occur in Egypt. The data have been extracted from published works from a variety of different sources, excluding all dubious and erroneous records. Information in the text is given in the following order: the present name of the taxon (the 
most recent combination) followed by the author name, year and page number of the original citation, the type locality, the reference in which the species is described in detail, geographical distribution (worldwide and Egypt) and the published works which mention Egyptian families.

The geographical distribution of each species is indicated by a three-letter abbreviation and given as presence in one or more of the following seven zoogeographical regions: (AFR) Afrotropical, (AUS) Australian, (NEA) Nearctic, (NEO) Neotropical, (ORI) Oriental, (PAC) Pacific and (PAL) Palearctic. Distribution in Egypt is indicated by a twoletter abbreviation and given as presence in one or more of the following six biogeographical zones: (CS) Northern coastal strip, (ED) Eastern Desert, (GE) Gebel Elba, (NV) Nile Valley and Delta, (SI) Sinai Peninsula and (WD) Western Desert (Fig. 1).

\section{RESULTS}

The following checklist contains 21 valid species from 13 genera of the families under consideration. Our study revealed that some species need to be confirmed or excluded from the species list of Egypt.

I. Family Helophoridae LEACH, 1815

Genus Helophorus FABRICIUS, 1775

Subgenus Rhopalohelophorus KUWERT, 1886

1-Helophorus (Rhopalohelophorus) angustatus MOTSCHULSKY, 1860

Helophorus angustatus MOTSCHULSKY, 1860:105

Type locality: Nile (Egypt).

Description: ANGUS (1992:90), FIKÁČEK (2009:142).

Geographical distribution: PAL - CS, NV, WD.

References: MotsChULSKY (1860), GEMMINGER \& HAROLD (1868), KUWERT (1885, 1886, 1890a), SAhlberg (1903a, 1930b), Zaitzev (1908), ANDres (1914), SAHLberg (1913), SHARP (1916), STOREY (1916), KNISCH (1924), ALFIERI (1976), ANGUS (1988, 1992), FAdL et al. (1993), SAlman (1996), Hebauer (1997a), HANSEn (1999, 2004), HebAuer (2006), FiKÁČEK (2009), FiKÁČEK et al. (2015), PRZEWOŹNy (2017). 


\section{Family Georissidae LAPORTE, 1840}

Genus Georissus LATREILLE, 1809

Subgenus Neogeorissus SATÔ, 1972

\section{2-Georissus (Neogeorissus) costatus LAPORTE, 1840}

Georissus costatus LAPORTE, 1840:45

Type locality: Spain.

Description: HEBAUER \& KLAUSNITZER (1998:14).

Geographical distribution: AFR - NV.

References: ALFIERI (1976), HANSEN (1999, 2004), HEBAUER (2006), MASCAGNi (2008), LITOVKIN \& FIKÁČEK (2011), FIKÁČEK \& PRZEWOŹNY (2015), PRZEWOŹNY (2017).

\section{3-Georissus (Neogeorissus) trifossulatus MOTSCHULSKY, 1843}

Georissus trifossulatus MOTSCHULSKY, 1843:658

Type locality: River Alazani (Georgia).

Description: FIKÁČEK \& FALAMARZI (2010:111).

Geographical distribution: PAL - CS.

References: ALFIERI (1976), HANSEN (1999, 2004), HEBAUER (2006), FIKÁČEK \& FALAMARZI (2010), FIKÁČEK \& PRZEWOŹNY (2015), PRZEWOŹNY (2017).

\section{Family Hydrochidae THOMSON, 1859}

Genus Hydrochus LEACH, 1817

\section{4-Hydrochus niloticus SHARP, 1903}

Hydrochus niloticus SHARP, 1903:9

Type locality: Gebel Ahmed Agha (Sudan).

Description: SHARP (1903:9), ORCHYMONT (1948:722).

Geographical distribution: AFR - Egypt (no locality data).

References: HEBAUER (1997a, 2006).

Remarks: HeBAUER (1997a, 2006) has reported the occurrence of H. niloticus in Egypt. The species was described by SHARP (1903) from several specimens collected from Sudan (thirteen specimens in a backwater near the White Nile, north of Gebel Ahmed Agha and two on water plants, Gebel Ahmed Agha). Many authors, such as ALFIERI (1976), FADL et al. (1993) and SALMAN (1996), mentioned the absence of this species from the Egyptian fauna depending on the original type locality. The species has an Afrotropical distribution, being known from Ethiopia, Mozambique and Sudan (HEBAUER 2006). 


\section{Family Spercheidae Erichson, 1837}

Genus Spercheus KugELANN, 1798

\section{5-Spercheus cerisyi GUÉRIN-MÉNEVILLE, 1842}

Spercheus cerisyi GUÉRIN-MÉNEVILLE, 1842:71

Type locality: Alexandria (Egypt).

Description: HEBAUER (1997b:36), DARILMAZ \& KIYAK (2011:442).

Geographical distribution: AFR, PAL - CS, ED, NV.

References: GEMMINGER \& HAROLD (1868), MARSEUL (1882), KUWERT (1890a), FAIRMAIRE (1892), SAHLBERG (1903a, 1930b), ZAITZEV (1908), STOREY (1916), KNISCH (1924), ORCHYMONT (1927), ALFIERI (1976), El SHERIF et al. (1976), YANO et al. (1983), FADL et al. (1993), SALMAN (1996), HEBAUER (1997a, 1997b), HANSEN (1999, 2004), HENDAWY et al. (2005), VONDEL (2005), HEBAUER (2006), ZALAT et al. (2008), DARILMAZ \& KIYAK (2011), BADAWY et al. (2013), FIKÁČEK (2015), PRZEWOŹNY (2017).

\section{6-Spercheus emarginatus (SCHALLER, 1783)}

Dytiscus emarginatus SCHALLER, 1783:327

Type locality: Germany.

Description: HEBAUER (1997b:17).

Geographical distribution: PAL - CS, NV.

References: FADL et al. (1993), SALMAN (1996), HEBAUER (2006).

\section{7-Spercheus platycephalus interruptus FAIRMAIRE, 1892} Spercheus interruptus FAIRMAIRE, 1892:88

Type locality: Obock (Djibouti).

Description: HEBAUER (1997b:19).

Geographical distribution: AFR, PAL - Egypt (No locality data).

References: HebAUER (1997b), HANSEN (1999, 2004), HeBAUER (2006), FiKÁČEK (2015), PRZEWOŹNY (2017).

\section{Family Scirtidae FLEMING, 1821}

Subfamily Scirtinae FLEMING, 1821

Genus Contacyphon GozIS, 1886

\section{8-Contacyphon laevipennis (TOURNIER, 1868)}

Cyphon laevipennis TOURNIER, 1868:64

Type locality: Jura, dans le Val d'Arderan (Switzerland). 
Description: KLAUSNITZER (2009:261).

Geographical distribution: PAL - ED, NV.

References: KLAUSNITZER (1991, 1997, 2006, 2009, 2016a, 2016b).

\section{Family Elmidae CURTIS, 1830}

Subfamily Elminae CURTIS, 1830

Tribe Elmini CURTIS, 1830

Subtribe Elmina CURTIS, 1830

Genus Oulimnius GozIs, 1886

9-Oulimnius aegyptiacus (KUWERT, 1890b)

Limnius aegyptiacus KUWERT, 1890b:44

Type locality: Egypt.

Description: BERTHÉLEMY (1979:74).

Geographical distribution: PAL - SI.

References: Kuwert (1890b), Peyerimhoff (1907), ZAITZeV (1908, 1910), ALFieri (1976), JäCH et al. (2006), JÄCH \& KODADA (2016), JÄCH et al. (2016).

Subfamily Larainae LECONTE, 1861

Tribe Potamophilini MuLSANT \& REY, 1872

Genus Potamodytes GrouvelLe, 1896a

\section{0-Potamodytes subrotundatus PIC, 1939}

Potamodytes subrotundatus PIC, 1939:143

Type locality: Egypt.

Description: PIC (1939:143).

Geographical distribution: AFR, PAL - NV.

References: PIC (1939), ALFIERI (1976), JÄCH et al. (2006), JÄCH \& KODADA (2016), JÄCH et al. (2016).

\section{Family Dryopidae BILLBERG, 1820}

Genus Dryops OLIVIER, 1791

\section{1-Dryops gracilis (KARSCH, 1881)}

Parnus gracilis KARSCH, 1881:45

Type locality: Ain Schersozura (Libya).

Description: OLMI (1976:58).

Geographical distribution: AFR, PAL - SI. 
References: BOLlOW (1938), KODADA \& JÄCH (2006, 2016), MASCAGNI et al. (2016).

\section{2-Dryops luridus (ERICHSON, 1847)}

Parnus luridus ERICHSON, 1847:513

Type locality: Schlesien, Hard (Holland).

Description: OLMI (1976:60).

Geographical distribution: AFR, PAL - NV, SI.

References: PEYERIMHOFF (1907), KNEUCKER (1922), ALFIERI (1976), ZALAT et al. (2008), KODADA \& JÄCH $(2006,2016)$.

\section{3-Dryops rufipes (KRYNICKI, 1832)}

Parnus rufipes KRYNICKI, 1832:115

Type locality: Kharkov (Ukraine).

Description: OLMI (1976:73).

Geographical distribution: PAL - Egypt (No locality data).

References: BOLLOW (1938), OLMI (1976), KODADA \& JÄCH (2006), BOUKAL et al. (2007), TAŞAR (2014), KODADA \& JÄCH (2016), MASCAGNI et al. (2016).

Genus Parahelichus LÖBL \& SMETANA, 2006

\section{4-Parahelichus fenyesi (REITTER, 1894)}

Dryops fenyesi REITTER, 1894:313

Type locality: Helwan (Egypt).

Description: BoLlow (1940:28) “as Helichus (Parahelichus) fenyesi REITTER, 1894".

Geographical distribution: AFR, PAL - NV.

References: ReITTER (1894), ZAITZEV (1908, 1910), Bollow (1940), AlfiERI (1976), OLMI (1976), KODADA \& JÄCH (2006, 2016).

\section{Family Limnichidae ERICHSON, 1846}

Subfamily Limnichinae ERICHSON, 1846

Genus Bothriophorus MULSANT \& REY, 1852

\section{5-Bothriophorus atomus MULSANT \& REY, 1852}

Bothriophorus atomus MULSANT \& REY, 1852:20

Type locality: Europe (SPANGLER et al. 2001).

Description: HERNANDO et al. (1999:39).

Geographical distribution: PAL - CS. 
References: AlFiERI (1976), RATTI (1983), HERNANDO \& RIBERA (2006, 2016b), LITOVKIN \& SAZHNEV (2016).

Genus Pelochares Mulsant \& REY, 1869

\section{6-Pelochares versicolor (WALTL, 1838)}

Limnichus versicolor WALTL, 1838:273 (not page no. 274 as stated by HERNANDO and RIBERA 2006, 2016b).

Type locality: New Caledonia (Europe) (SPANGLER et al. 2001).

Description: GANGLBAUER (1904:52).

Geographical distribution: PAL - NV.

References: ALFIERI (1976), HERNANDO \& RIBERA (2006), BOUKAL et al. (2007), ZINETTI \& TERZANI (2009), HERNANDO \& RIBERA (2016b).

\section{Family Heteroceridae MACLEAY, 1825}

Subfamily Heterocerinae MACLEAY, 1825

Tribe Augylini PACHECO, 1964

Genus Augyles SCHIÖDTE, 1866

\section{7-Augyles (Augyles) niloticus (GROUVELLE, 1896b)}

Heterocerus niloticus GROUVELLE, 1896b:54

Type locality: Luxor (Egypt).

Description: CHARPENTIER (1965:285).

Geographical distribution: AFR, PAL - NV.

References: Grouvelle (1896b), SAHLBERG (1903a, 1903b), ZAITZeV (1908, 1910), Andres (1914), SAHLberg (1913), WinkLer (1932), Charpentier (1965), Hanna (1969a, 1969b, 1969c), ALFIERI (1976), SKALICKÝ (1999), SKALICKÝ et al. (2002), MASCAGNI (2006), MASCAGNI \& MONTE (2008, 2009), MASCAGNI $(2014,2016)$.

Tribe Heterocerini MACLEAY, 1825

Genus Heterocerus FABRICIUS, 1792

\section{8-Heterocerus fenestratus (THUNBERG, 1784)}

Dermestes fenestratus THUNBERG, 1784:3

Type locality: Sweden.

Description: AGUILERA et al. (1998:85), MASCAGNI (2014:93).

Geographical distribution: NEA, ORI, PAL - CS, NV, SI. 
References: ALFIERI (1976), SKALICKÝ (2001), SKALICKÝ et al. (2002), MASCAGNI $\&$ MonTe $(2008,2009)$.

Remarks: SKALICKÝ (2001) examined some Egyptian specimens from H. fenestratus from Port Said, housed in the Hungarian Natural History Museum, Budapest (TMB). The Egyptian distribution was not included in the catalogues of MASCAGNI $(2006,2016)$. In North Africa, the species is known from Algeria, Morocco and Tunisia (MASCAGNI 2016).

\section{9-Heterocerus flexuosus STEPHENS, 1828}

Heterocerus flexuosus STEPHENS, 1828:101

Type locality: The banks of the Thames, beyond Gravesend (England).

Description: CHARPENTIER (1965:280), MASCAGNI (2014:91).

Geographical distribution: AFR, PAL - ED.

References: SKalickÝ (2001), SKalickÝ et al. (2002), MASCAGNI (2006), MASCAGNI $\&$ MONTE $(2008,2009)$, MASCAGNI $(2014,2016)$.

\section{0-Heterocerus medius CHARPENTIER, 1965}

Heterocerus medius CHARPENTIER, 1965:238

Type locality: Cairo Pyramids (Egypt).

Description: CHARPENTIER (1965:238).

Geographical distribution: AFR, PAL - NV.

References: CHARPENTIER (1965), SKALICKÝ et al. (2002), MASCAGNI (2006), MASCAGNI $\&$ MonTE $(2008,2009)$, MASCAGNI $(2014,2016)$.

\section{1-Heterocerus thebaicus GROUVELLE, 1896b}

Heterocerus thebaicus thebaicus GROUVELLE, 1896b:55

Type locality: Luxor (Egypt).

Description: CHARPENTIER (1965:239).

Geographical distribution: AFR, PAL - NV.

References: Grouvelle (1896b), Zaitzev (1908, 1910), WinKLER (1932), CharPentier (1965), Alfieri (1976), SKALICKÝ et al. (2002), MASCAGNI (2006), MASCAGNI \& Monte $(2008,2009)$, MASCAGNI $(2014,2016)$. 


\section{Species excluded from the checklist or needing confirmation}

\section{-Helophorus (Rhopalohelophorus) lapponicus THOMSON, 1853:42}

HEBAUER (1997a) reported a doubtful record of H. lapponicus from Egypt. The species is entirely absent from Africa (HANSEN 1999, FIKÁČEK et al. 2015, PRZEWOŹNY 2017) and its presence in Egypt needs confirmation.

\section{-Helophorus (Rhopalohelophorus) pallidipennis MULSANT \& WACHANRU, 1852:6}

The distribution of $H$. pallidipennis has been previously reported in Egypt by many authors, such as ORCHYMONT (1927), AlFIERI (1976), SALMAN (1996) and HEBAUER (1997a). The species is entirely absent from Africa (FIKÁČEK et al. 2015, PRZEWOŹNY 2017), and the previous records of this species from Egypt need confirmation (HANSEN 1999).

\section{-Helophorus (Rhopalohelophorus) mervensis SEMENOv, 1900:587}

HEBAUER (1997a) has reported a doubtful record of H. mervensis from Egypt. The species is entirely absent from Africa (HANSEN 1999, FIKÁČEK et al. 2015, PRZEWOŹNY 2017), and its presence in Egypt needs confirmation.

\section{-Helophorus (Trichohelophorus) oscillator (SHARP, 1915:163)}

The distribution of $H$. oscillator has been doubtfully reported in Egypt by many authors, such as KNisCh (1924), Alfieri (1976), ANGUS (1992), FADL et al. (1993), HebaUER (1994), HANSEN (1999) and HEBAUER (2006). SHARP (1915) described H. oscillator as probably being from Egypt. He was uncertain, as the type had become separated from its card (ANGUS 1992). If not from Egypt, it is from Mesopotamia or the Hedjaz (SHARP 1915). As explained by ANGUS (1984), this is a little known species, which appears to be dispersed in Israel, Syria and Turkey (FIKÁČEK et al. 2015, PRZEWOŹNY 2017).

\section{CONCLUSION}

In Egypt, with its distinctly different regions and different environments, the number of genera and species are likely to be much higher than has been recorded so far. More intensive studies are required on these families. 


\section{REFERENCES}

Aguilera P., Mascagni A., Ribera I. 1998. The family Heteroceridae MacLeay, 1825 (Coleoptera, Dryopoidea) in the Iberian Peninsula and Balearic Islands. Miscelánea Zoológica 21(1): 75-100.

ALfiERI A. 1976. Coleoptera of Egypt. Mémoires de la Société Entomologique d'Egypte 5: i-xvi, $1-361$.

ANDRES A. 1914. Note Bibliographique; Coleoptera mediterranea orientalia, quae in Aegypto, Palaestina, Syria, Caramania atque in Anatolia occidentali anno 1904 collegerunt John SAHLBERG et Unio SAALAS. Bulletin de la Société Entomologique d'Égypte 3: 84-132.

ANGUS R.B. 1988. Notes on the Helophorus (Coleoptera, Hydrophilidae) occurring in Turkey, Iran and neighbouring territories. Revue Suisse de Zoologie 95(1): 209-248.

ANGus R.B. 1992. Insecta Coleoptera Hydrophilidae Helophorinae. [in:] J. SCHwoERBEL, P. ZwICK (eds.). Süsswasserfauna von Mitteleuropa 20/10-2. Gustav Fischer Verlag, Stuttgart - Jena - New York, i-xi + 1-144.

ANGus R.B. 1984. Towards a revision of the Palearctic species of Helophorus F. (Coleoptera, Hydrophilidae) I. Entomological Review 63(3): 89-119.

ARChAngelsky M. 2001. A new Neotropical species of Spercheus KLUGELANN, and its larval stages (Coleoptera, Hydrophiloidea: Spercheidae). Studies on Neotropical Fauna and Environment 36(3): 199-204

Archangelsky M., Beutel R.G., KomareK A. 2016. 12. Hydrophiloidea Latreille. 1802. [in:] R.G. Beutel, R.A.B. LesChen (eds.). Handbook of Zoology. Arthropoda: Insecta Coleoptera, Beetles. Volume 1: Morphology and Systematics (Archostemata, Adephaga, Myxophaga, Polyphaga partim), $2^{\text {nd }}$ edition. Walter de Gruyter GmbH, Berlin - Boston, 231-272.

Badawy R.M., El Hoseny I., Talal M. 2013. Biodiversity and Seasonal Fluctuation of Aquatic and Semiaquatic Insects in Rashid Stream, Kafr El Zayat (Gharbyia Governorate). Egyptian Academic Journal of Biological Sciences (Entomology) 6(1): 47-66.

BAHA El Din S.M. 2001. Egypt. [in:] L.D.C. FishPOOL, M.I. Evans (eds.). Important bird areas in Africa and associated islands: Priority sites for conservation. Pisces Publications and Birdlife International, Newbury and Cambridge, UK, 241-264.

BERTHÉLEMY C. 1979. Elmidae de la région paléarctique occidentale: systématique et répartition (Coleoptera Dryopoidea). Annales de Limnologie 15(1): 1-102.

Beutel R.G. 2016. 19 Byrrhoidea LAtreille, 1804. [in:] R.G. Beutel, R.A.B. Leschen (eds.) Handbook of Zoology Arthropoda: Insecta Coleoptera, Beetles Volume 1: Morphology and Systematics (Archostemata, Adephaga, Myxophaga, Polyphaga partim), $2^{\text {nd }}$ edition. Walter de Gruyter GmbH, Berlin - Boston, 553-554.

BILlBerg G.J. 1820. Enumeratio Insectorum in Museo Gust. Joh. BILlBerg. Typus Gadelianus, Stockholm.

Bollow H. 1938. Monographie der palaearktischen Dryopidae, mit Berücksichtigung der eventuell transgredierenden Arten (Coleoptera). Mitteilungen der Münchener Entomologischen Gesellschaft 28(3): 319-371. 
BolLOw H. 1940. Monographie der palaearktischen Dryopidae, mit Berücksichtigung der eventuell transgredierenden Arten. (Coleoptera). Mitteilungen der Münchener Entomologischen Gesellschaft 30(1): 24-71.

Boukal D.S., Boukal M., FikÁČEK M., HáJeK J., KleČKa J., SkalickÝ S., ŠŤASTnÝ J., TrávníČEK D. 2007. Katalog vodních brouků České republiky. Catalogue of water beetles of the Czech Republic (Coleoptera: Sphaeriusidae, Gyrinidae, Haliplidae, Noteridae, Hygrobiidae, Dytiscidae, Helophoridae, Georissidae, Hydrochidae, Spercheidae, Hydrophilidae, Hydraenidae, Scirtidae, Elmidae, Dryopidae, Limnichidae, Heteroceridae, Psephenidae). Klapalekiana 43 (Suppl.): 1-289.

Charpentier R. 1965. A monograph of the family Heteroceridae (Coleoptera) of the Ethiopian Region. [in:] B. HANSTRÖM, P. BRINCK, G. RUDEBECK (eds.). South African Animal Life. Results of the Lund University Expedition in 1950-1951. Vol. XI. Almqvist and Wiksell, Stockholm, 214-343.

CURTIS J. 1830. British entomology; being illustrations and descriptions of the genera of insects found in Great Britain and Ireland: containing coloured figures from nature of the most rare and beautiful species, and in many instances of the plants upon which they are found. Vol. VII. Curtis, London.

Darilmaz M.C., KiYAK S. 2011. A study on the family Spercheidae (Coleoptera) from Turkey. Turkish Journal of Zoology 35(3): 441-444.

EL SHERIF S.I., ISA A.L., LUTFALlaH A.F. 1976. Survey of aquatic insects in rice nurseries and fields. Agricultural Research Review 54(1): 93-98.

ERICHSON W.F. 1837. Die Käfer der Mark Brandenburg. Erster Band, Erste Abtheilung. Morin, Berlin.

ERICHSON W.F. 1846. Naturgeschichte der Insecten Deutschlands. Erste Abtheilung. Coleoptera. Dritter Band, Teil 2. Nicolaische Buchhandlung, Berlin.

ERICHSON W.F. 1847. Naturgeschichte der Insecten Deutschlands. Erste Abtheilung. Coleoptera. Dritter Band, Teil 3. Nicolaische Buchhandlung, Berlin.

FABRICIUS J.C. 1775. Systema entomologiae, sistens insectorum classes, ordines, genera, species, adiectis synonymis locis, descriptionibus, observationibus. Libraria Kortii, Flensburgi et Lipsiae.

FABRICIUS J.C. 1792. Entomologia systematica emendata et aucta. Secundum classes, ordines, genera, species adiectis, synonimis, locis, observationibus, descriptionibus. Tom. I. Christ. Gottl. Proft, Hafniae.

FAdL H.H., El SheriF L.S., ShaARAwi F.A., SALMAN A.Y. 1993. Hydrophilidae of Egypt (subfamilies Helophorinae, Hydrochinae and Spercheinae) Coleoptera. Bulletin of the Entomological Society of Egypt 71: 101-108.

FAIRMAIRE L. 1892. Coléoptères d'Obock. Troisieme partie. Revue d'Entomologie 11(1): 77-127.

FIKÁČEK M. 2009. Order Coleoptera, family Helophoridae. [in:] A. VAN HARTEN (ed.). Arthropod fauna of the UAE. Volume 2. UAE Insect Project, Sharjah, United Arab Emirates: 142-144.

FIKÁČEK M. 2015. Spercheidae. [in:] I. LÖBL, D. LÖBL (eds.). Catalogue of Palaearctic Coleoptera, vol. 2/1. Revised and updated version. Hydrophiloidea - Staphylinoidea. Brill, Leiden - Boston, $36-37$. 
FikÁČEK M., Angus R.B., Gentili E., Jia F., Minoshima Y.N. 2015. Helophoridae. [in:] I. LöBl, D. LÖBL (eds). Catalogue of Palaearctic Coleoptera, vol. 2/1. Revised and updated version. Hydrophiloidea - Staphylinoidea. Brill, Leiden - Boston, 25-33.

FiKÁČEK M., FAlAMARZI S. 2010. Georissus persicus sp. nov. from Iran, with notes on the WestPalaearctic species of the G. laesicollis group (Coleoptera: Georissidae). Acta Entomologica Musei Nationalis Pragae 50(1):107-116.

FIKÁČEK M., PRZEwoźNY M. 2015. Georissidae. [in:] I. LÖBL, D. LÖBL (eds). Catalogue of Palaearctic Coleoptera, vol. 2/1. Revised and updated version. Hydrophiloidea - Staphylinoidea. Brill, Leiden - Boston, 33-35.

FIKÁČEK M., TRÁVNÍČEK D. 2009. Order Coleoptera, family Georissidae. [in:] A. vAN HARTEN (ed.). Arthropod fauna of the UAE. Volume 2. UAE Insect Project, Sharjah, United Arab Emirates: $145-148$.

FLEMING J. 1821. Insecta. Supplementa to the fourth, fifth, and sixth editions of the Encyclopaedia Britannica, with preliminary dissertation on the history of the sciences. Volume 5, Part 1. Archibald Constable and Company, Edinburg.

Ganglbauer L. 1904. Die Käfer von Mitteleuropa. Die Käfer der osterreichisch-ungarischen Monarchie, Deutschlands, der Schweiz, sowie des franzosischen und italienischen Alpengebietes. Vierter Band, erste Hälfte. Karl Gerold's Sohn, Wien.

GEMminger M., HAROLD E. vON 1868. Catalogus Coleopterorum hucusque descriptorum synonymicus et systematicus. Tom. II. Dytiscidae, Gyrinidae, Hydrophilidae, Staphylinidae, Pselaphidae, Gnostidae, Paussidae, Scydmaenidae, Silphidae, Trichopterygidae, Scaphidiidae. E.H. Gummi, Monachii.

GOZIS M. DES 1886. Recherche de l'espèce typique de quelques anciens genres. Rectifications synonymiques et notes diverses. Herbin, Montluçon.

Grouvelle A. 1896a. Note sur les subdivisions génériques des potamophiliens (Col.). Bulletin de la Société Entomologique de France 1896: 77-79.

Grouvelle A. 1896b. Potamophilides, dryopides, helmides et heterocerides des Indes Orientales. Annali del Museo Civico di Storia Naturale di Genova Serie II 17(XXXVII): 32-56.

GUÉRIN-MÉNEVILLE F.E. 1842. [in:] Iconographie du règne animal de G. CUVIER, ou représentation d'après nature de l'une des espèces les plus remarquables et souvent non figurées de chaque genre d'animaux. Avec un texte descriptif mis au courant de la science. Ouvrage pouvant servir d'atlas à tous les traités de zoologie. II. Planches des animaux invertébrés. Insectes. J.B. Bailliere, Paris, $71-73$.

HANNA H.M. 1969a. Effect of weather conditions on flight activity of nocturnal Coleoptera. Bulletin de la Société Entomologique d'Egypte 53: 205-219.

HANNA H.M. 1969b. Effect of lunar periodicity on flight activity of Coleoptera. Bulletin de la Société Entomologique d'Egypte 53: 473-482.

HanNa H.M. 1969c. Studies on catches of Coleoptera in light trap, at Assiut. Bulletin de la Société Entomologique d'Egypte 53: 591-613.

HANSEN M. 1999. World Catalogue of Insects. Hydrophiloidea (Coleoptera). Vol. 2. Apollo Books, Stenstrup. 
Hansen M. 2004. Family Hydrophilidae. [in:] I. LÖBL, A. Smetana (eds.). Catalogue of Palaearctic Coleoptera Volume 2. Hydrophilidae - Histeroidea - Staphylinoidea. Apollo Books, Stenstrup, 44-68.

Hebauer F. 1994. The Hydrophiloidea of Israel and the Sinai. Zoology in the Middle East 10(1): 73-137.

HEBAUER F. 1997a. Annotated checklist of Hydrophilidae and Helophoridae (Insecta: Coleoptera) of the Arabian Peninsula with a description of a new genus and species. Fauna of Saudi Arabia 16: $255-276$.

Hebauer F. 1997b. Revision der Arten der Familie Spercheidae ERIChson, 1837 (Coleoptera, Hydrophiloidea). Entomologische Blätter für Biologie und Systematik der Käfer 93(1): 9-42.

Hebauer F. 2006. Checklist of the Hydrophiloidea of Africa and adjacent archipelagos (Coleoptera: Epimetopidae, Georissidae, Helophoridae, Hydrochidae, Hydrophilidae, Spercheidae). Entomologische Zeitschrift 116(1): 19-36.

Hebauer F., KLAuSNitzer B. 1998. Süsswasserfauna von Mitteleuropa 20/7, 8, 9, 10-1. Insecta: Coleoptera: Hydrophiloidea (Exkl. Helophorus). Gustav Fischer Verlag, Stuttgart - Jena Lübeck - Ulm.

Hendawy A.S., Sherif M.R., Abada A.E., El-Habashy M.M. 2005. Aquatic and semi-aquatic insects occurring in the Egyptian rice fields and hazardous effect of insecticides. Egyptian Journal of Agricultural Research 83(5B): 493-502.

Hernando C., Aguilera P., Ribera I. 1999. Bothriophorus atomus Mulsant \& Rey, 1852 nuevo para la península Ibérica (Coleoptera, Limnichidae). Orsis 14: 39-41.

HeRnANDo C., Ribera I. 2006. Family Limnichidae ERICHSON, 1847. [in:] I. LÖBl A. SMETANA (eds.) Catalogue of Palaearctic Coleoptera, Volume 3, Scarabaeoidea - Scirtoidea - Dascilloidea Buprestoidea - Byrrhoidea. Apollo Books, Stenstrup, 443-446.

HeRnANDo C., Ribera I. 2016a. 19.5 Limnichidae ERIChSON, 1846. [in:] R.G. BEutel, R.A.B. LESCHEN (eds). Handbook of Zoology. Arthropoda: Insecta Coleoptera, Beetles. Volume 1: Morphology and Systematics (Archostemata, Adephaga, Myxophaga, Polyphaga partim), $2^{\text {nd }}$ edition. Walter de Gruyter GmbH, Berlin/ Boston, 605-612.

Hernando C., Ribera I. 2016b. Family Limnichidae ERICHSON, 1846. [in:] I. LÖBL, D. LÖBL (eds). Catalogue of Palaearctic Coleoptera. Volume 3, Scarabaeoidea, Scirtoidea, Dascilloidea, Buprestoidea, Byrrhoidea: Revised and updated edition, Brill, Leiden-Boston, 607-610.

HoAth R. 2003. A field guide to the mammals of Egypt. The American University in Cairo Press, Egypt.

JäCH M.A. 1998. Annotated check list of aquatic and riparian/littoral beetle families of the world (Coleoptera). [in:] M.A. JÄCH, L. LI (eds.). Water Beetles of China, Vol. II. ZoologischBotanische Gesellschaft in Österreich and Wiener Coleopterologenverein, Wien, 25-42.

JÄCH M.A. KodAdA J., BROJER M., ShePARD W.D., ČIAMPOR F.JR. 2016. World Catalogue of Insects. Volume 14. Coleoptera: Elmidae and Protelmidae. Brill Academic Publishers, Leiden.

JÄCH M.A., KodAdA J. 2016. Family Elmidae CurTis, 1830. [in:] I. LÖBL, D. LöBL (eds.). Catalogue of Palaearctic Coleoptera. Volume 3, Scarabaeoidea, Scirtoidea, Dascilloidea, Buprestoidea, Byrrhoidea: Revised and updated edition. Brill, Leiden - Boston, 591-603. 
Jäch M.A., Kodada J., ČIAMPOR F. 2006. Family Elmidae Curtis, 1830. [in:] I. LöBl, A. Smetana (eds.). Catalogue of Palaearctic Coleoptera. Volume 3, Scarabaeoidea - Scirtoidea - Dascilloidea - Buprestoidea - Byrrhoidea. Apollo Books, Stenstrup, 432-440.

KARSCH F. 1881. Die Käfer der RoHLFS'schen Afrikanischen Expedition 1878-79. Berliner Entomologische Zeitschrift 25(1): 41-50, pl. II.

KLAUSNItZER B. 1991. Über die Helodidae der östlichen und südlichen Mediterraneis. Koleopterologische Rundschau 61: 159-170.

KLAusNiTZER B. 1997. Scirtiden-Funde aus Nordafrika und Kreta (Col., Scirtidae). Entomologische Nachrichten und Berichte 41: 206-207.

KLausnitzer B. 2006. Family Scirtidae Fleming, 1821. [in:] I. LöBl, A. Smetana (eds.). Catalogue of the Palaearctic Coleoptera. Volume 3, Scarabaeoidea - Scirtoidea - Dascilloidea Buprestoidea - Byrrhoidea. Apollo Books, Stenstrup, 316-323.

KLAusnitZer B. 2009. Süßwasserfauna von Mitteleuropa, 20/17. Insecta: Coleoptera: Scirtidae. Spektrum Akademischer Verlag, Heidelberg.

KLAusnitzer B. 2016a. Family Scirtidae Fleming, 1821. [in:] I. LÖBL, D. LöBl (eds). Catalogue of Palaearctic Coleoptera. Volume 3, Scarabaeoidea, Scirtoidea, Dascilloidea, Buprestoidea, Byrrhoidea: Revised and updated edition. Brill, Leiden - Boston, 412-425.

KLAuSNITZER B. 2016b. Verbergen sich unter Contacyphon laevipennis (TourniER, 1868) (Coleoptera, Scirtidae) zwei Arten? (207. Beitrag zur Kenntnis der Scirtidae). Linzer biologische Beiträge 48(1): 551-558.

KNEUCKER J.A. 1922. Zoologische Ergebnisse zweier in den Jahren 1902 und 1904 durch die Sinaihalbinsel unternommener botanischer Studienreisen. II. Teil. Entomologische Blätter 18 (1-3): 20-28.

KNISCH A. 1924. Coleopterorum Catalogus 14(79). Hydrophilidae. W. Junk, Berlin.

KodAda J., Jäch M.A. 2006. Family Dryopidae Bilberg, 1820. [in:] I. LöBl, A. SMETANA (eds.). Catalogue of Palaearctic Coleoptera. Volume 3, Scarabaeoidea - Scirtoidea - Dascilloidea Buprestoidea - Byrrhoidea. Apollo Books, Stenstrup, 441-443.

Kodada J., Jäch M.A. 2016. Family Dryopidae BILlberg, 1820. [in:] I. LöBl, D. LöBl (eds.). Catalogue of Palaearctic Coleoptera. Volume 3, Scarabaeoidea - Scirtoidea - Dascilloidea Buprestoidea - Byrrhoidea: Revised and updated edition. Brill, Leiden-Boston, 591-603.

KodAdA J., JäCH M.A., ČIAMPOR F. Jr. 2016. 19.3 Dryopidae BILBERG, 1820 (1817). [in:] R.G. BEUTEL, R.A.B. LESCHEN (eds.). Handbook of Zoology. Arthropoda: Insecta Coleoptera, Beetles. Volume 1: Morphology and Systematics (Archostemata, Adephaga, Myxophaga, Polyphaga partim), $2^{\text {nd }}$ edition. Walter de Gruyter GmbH, Berlin - Boston, 590-602.

KRYNICKI J. 1832. Enumeratio Coleopterorum Rossiae meridionalis et praecique in Universitatis Caesareae Charkoviensis circulo obvenientium, quae annorum 1827-1831 spatio observavit, P.P.E. Ioannes KRYNICKI. Bulletin de la Société impériale des naturalistes de Moscou 5: 65-179, pls II-III.

KugELann J.G. 1798. [new taxa]. [in:] J.C.W. Illiger (ed.). Verzeichniss der Käfer Preussens. Entworfen von Johann Gottlieb KugelanN, Apotheker in Osterode. Mit einer Vorrede des Professors und Pagenhofmeister HeLlwIG in Braunschweig, und dem angehangten Versuche einer natürlichen Ordnungs- und Gattungs-Folge der Insekten. J.J. Gebauer, Halle. 
KUwERT A. 1885. Beiträge zur Kenntniss der Helophoren aus Europa und den angrenzenden Ländern. Wiener Entomologische Zeitung 4: 261-264.

KUwERT A. 1886. General-Uebersicht der Helophorinen Europas und der angrenzenden Gebiete. Wiener Entomologische Zeitung 5: 247-250.

KUwERT A. 1890a. Bestimmungs-Tabellen der europäischen Coleopteren. XX Heft. Hydrophilidae. II. Abteilung: Sphaeridiini und Helophorini. Verhandlungen des Naturforschenden Vereins in Brünn 28: 159-328.

Kuwert A. 1890b. Bestimmungstabelle der Parniden Europas, der Mittelmeerfauna, sowie der angrenzenden Gebiete. Verhandlungen der Kaiserlich-Königlichen Zoologisch-Botanischen Gesellschaft in Wien 40: 15-54.

LAPORTE [= CASTElnau], F.L.N. CAumont DE 1840. Histoire naturelle des insectes coléoptères. Tome deuxieme. In: Histoire naturelle des animaux articules, annelides, crustaces, arachnides, myriapodes et insectes. Tome troisieme. P. Duménil, Paris.

LATREILLE P.A. 1809. Genera crustaceorum et insectorum secundum ordinem naturalem in familias disposita, iconibus exemplisque plurimis explicata. Tomus quartus et ultimus. A. Koenig, Parisiis.

Lawrence J.F. 2016a. Scirtoidea Fleming, 1821. [in:] R.G. Beutel, R.A.B. Leschen (eds.). Handbook of Zoology. Arthropoda: Insecta Coleoptera, Beetles. Volume 1: Morphology and Systematics (Archostemata, Adephaga, Myxophaga, Polyphaga partim), $2^{\text {nd }}$ edition. Walter de Gruyter GmbH, Berlin - Boston, 204-206.

LAwrence J.F. 2016b. 10.4 Scirtidae Fleming, 1821. [in:] R.G. Beutel, R.A.B. LesChen (eds.) Handbook of Zoology. Arthropoda: Insecta Coleoptera, Beetles. Volume 1: Morphology and Systematics (Archostemata, Adephaga, Myxophaga, Polyphaga partim), $2^{\text {nd }}$ edition. Walter de Gruyter GmbH, Berlin - Boston, 215-225.

LEACH W.E. 1815. Entomology. [in:] D. BREwSTER (ed.). The Edinburgh Encyclopaedia. Volume 9. Balfour, Edinburgh, 57-172.

LEACH W.E. 1817. The Zoological Miscellany, being descriptions of new or interesting animals. Vol. III. R.P Nodder, London.

LeConte J.L. 1861. Smithsonian Miscellaneous Collections 3. Classification of the Coleoptera of North America. Part 1. Smithsonian Institution, Washington.

Litovkin S.V., FIKÁČEK M. 2011. New records of Georissus costatus LAPORTE DE CASTELNAU, 1840 (Coleoptera: Georissidae) from Russia. Russian Entomological Journal 20(4): 383-385.

Litovkin S.V., SaZhnev A.S. 2016. New data on the distribution and biology of water beetles (Coleoptera: Haliplidae, Dytiscidae, Helophoridae, Georissidae, Hydrophilidae, Limnichidae, Curculionidae) in Russia. Euroasian Entomological Journal 15(1): 17-24. (In Russian)

Löbl I., Smetana A. 2006. New acts and comments. Dryopidae. [in:] I. LöBl, A. Smetana (eds). Catalogue of Palaearctic Coleoptera. Volume 3, Scarabaeoidea - Scirtoidea - Dascilloidea Buprestoidea - Byrrhoidea. Apollo Books, Stenstrup, 61.

MacLEay W.S. 1825. Annulosa Javanica, or an attempt to illustrate the natural affinities and analogies of the insects collected in Java by Thomas HoRSFIELD, M.D.F.L. and G.S. and deposited by him in the museum of the honourable East-India Company. Number 1. Kingsbury, Parbury, and Allen, London. 
MARSEUL S.A. DE 1882. Nouveau répertoire contenant les descriptions des espèces de coléoptères de l'ancien-monde publiées isolément ou en langues étrangères, en dehors des monographies ou traités spéciaux et de l'Abeille. L'Abeille 20: 1-196.

Mascagni A. 2006. Family Heteroceridae MacLeay, 1825. [in:] I. Löbl, A. Smetana (eds.) Catalogue of Palaearctic Coleoptera, volume 3, Scarabaeoidea - Scirtoidea - Dascilloidea Buprestoidea - Byrrhoidea. Apollo Books, Stenstrup, 446-449.

MASCAGNi A. 2008. Nuovi dati su tre specie di Georissidae paleartici (Coleoptera). Onychium 6: $62-63$.

MASCAGNI A. 2014. The variegated mud-loving beetles of Europe (first part) (Coleoptera: Heteroceridae). Onychium 10: 78-118.

MasCaGni A. 2016. Family Heteroceridae MACLEAy, 1825. [in:] I. LöBl, D. LöBL (eds). Catalogue of Palaearctic Coleoptera. Volume 3, Scarabaeoidea - Scirtoidea - Dascilloidea - Buprestoidea Byrrhoidea: Revised and updated edition. Brill, Leiden - Boston, 610-616.

Mascagni A., JäCh M.A., Ostovan H., GhahaRi H. 2016. Annotated checklist of Dryopidae and Heteroceridae (Coleoptera: Byrrhoidea) of Iran. Zootaxa 4144(3): 354-364.

Mascagni A., Monte C. 2008. Contributo alla conoscenza degli Heteroceridae del Nord Africa (Coleoptera). Onychium 6: 64-71.

Mascagni A., Monte C. 2009. Secondo aggiornamento alla checklist degli Heteroceridae della regione Afrotropicale (Coleoptera, Heteroceridae). Onychium 7: 61-72.

Motschulsky V. De 1843. Monographie du genre Georissus Latreille. Bulletin de la Société impériale des naturalistes de Moscou 16: 645-662, pls. xi-xii.

MotsChUlSKY V. DE 1860. Coléopteres rapportés de la Sibérie orientale et notamment des pays situés sur les bords du fleuve Amour par MM. Schrenck, Maack, Ditmar, Voznessenski etc. [in:] L. vON SCHRENCK (ed.). Reisen und Forschungen im Amur-Lande in den Jahren 1854-1856 im Auftrage der Kaiserl. Akademie der Wissenschaften zu St. Petersburg ausgefuhrt und in Verbindung mit mehreren Gelehrten herausgegeben. Band II. Zweite Lieferung. Coleopteren. Eggers \& Comp., St. Petersburg, 80-257, pls VI-XI.

Mulsant E., Rey C. 1852. Description d'un genre nouveau de la familie des byrrhiens. Annales de la Société Linnéenne de Lyon (N.S.) 1: 19-21.

Mulsant E., Rey C. 1869. Histoire naturelle des coléoptères de France. Piluliformes. Annales de la Société Linnéenne de Lyon (N.S.) 17: 201-378, 2 pls.

Mulsant E., Rey C. 1872. Histoire naturelle des Coléoptères de France. Improsternés, Unciferes, Diversicornes, Spinipedes. Deyrolle, Paris.

Mulsant E., Wachanru A. 1852. Premieres série de coléoptères nouveaux, ou peu connus. Memoires de l'Academie de Lyon, Classe des Sciences 2(2): 1-17.

Olivier A.G. 1791. Encyclopedie methodique, ou par ordre de matieres; par une societe de gens de lettres, de savans et d'artistes; Precedee d'un vocabulaire universel, servant de table pou l'Ouvrage, ornee des Portraits de MM. Diderot L'Alembert, premiers Editeurs de l'Encyclopedie. Histoire naturelle. Insectes. Tome sixieme. Pars I. Panckoucke, Paris.

Olmi M. 1976. Coleoptera: Dryopidae, Elminthidae. Fauna d'Italia. Vol. XII. Edizioni Calderini, Bologna. 
OrChymont A. 1927. Coléoptères Hydrophilides recueillis en Egypte. Bulletin de la Société Royale Entomologique d'Egypte, 11: 3-7.

OrChyMONT A. 1948. Report on the Palpicornia (Coleoptera), Mr. OMER-COOPER's Investigation of the Abyssinian Fresh Waters (Hugh Scотт Expedition). Proceedings of the Zoological Society of London 117(4): 716-741.

PACHECO M.F. 1964. Sistemática, filogenia y distribución de los heterocéridos de América (Coleoptera: Heteroceridae). Escuela Nacional de Agricultura, Colegio de Post-Graduados, Chapingo.

Peyerimhoff P.M. De Fontenelle 1907. Liste des Coléoptères du Sinai. L'Abeille 31: 1-48.

PIC M. 1939. Coléopteres nouveaux d'Egypte et du Sinai. Bulletin de la Société Fouad $1^{\text {er }}$ d'Entomologie 23: 143-149.

PrZewoźny M. 2017. Catalogue of Palearctic Hydrophiloidea (Coleoptera). Version 2017-01-01. Internet: http://www.waterbeetles.eu

RATTI E. 1983. Gli elementi caratteristici della coleotterofauna dei giuncheti alofili della Laguna di Venezia. Lavori della Società Veneziana di Scienze Naturali 8: 37-46.

ReitTer E. 1894. Uebersicht der Arten der Gattung Dryops Oliv. Wiener Entomologische Zeitung 13: 313 .

SAHLBERG J. 1903a. Entomologiska forskningsresor i Medelhafstrakterna och Centralasien företegna aren 1895-1896 samt 1898-1899. Resenberättelse. II. Resor i Palestina, Egypten, Tunisien och Algeriet. Öfversigt af Finska Vetenskaps-Societetens Förhandlingar 45(17): 1-39.

SAHLBERG J. 1903b. Coleoptera Levantina mensibus Februario et Martio in Palaestina et Aegypto inferiore collecta. Öfversigt af Finska Vetenskaps-Societetens Förhandlingar 45(18): 1-36.

SAHLBERG J. 1913. Coleoptera mediterranea orientalia, quae in Aegypto, Palaestina, Syria, Caramania atque in Anatolia occidentali anno 1904 collegerunt John SAHLBERG et Unio SAALAS. Öfversigt af Finska Vetenskaps-Societetens Förhandlingar (A) 55(19): 1-281.

SALAH M., RÉGIL J.A. 2014a. An annotated checklist of the aquatic Adephaga (Coleoptera) of Egypt I. Dytiscidae: Agabinae, Colymbetinae, Copelatinae, Dytiscinae and Laccophilinae. Boletín de la Sociedad Entomológica Aragonesa 54: 145-155.

SALAH M., RÉGIL J.A. 2014b. An annotated checklist of the aquatic Adephaga (Coleoptera) of Egypt II. Dytiscidae: Hydroporinae. Boletín de la Sociedad Entomológica Aragonesa 54: 293-305.

SALAh M., RÉGIL J.A. 2015. An annotated checklist of the aquatic Adephaga (Coleoptera) of Egypt III. Noteridae, Haliplidae and Gyrinidae. Zootaxa 4052(3): 187-200.

Salah M., RÉGIL J.A. in press. An Annotated Checklist of the Aquatic Polyphaga (Coleoptera) of Egypt II. Family Hydrophilidae. Coleopterists Bulletin (in press).

Salah M., Régil J.A., Valladares L.F. 2014. An annotated checklist of the aquatic Polyphaga (Coleoptera) of Egypt I. Family Hydraenidae. Zootaxa 3873(3): 275-284.

Salman A.Y.A.M. 1996. Survey and taxonomic study of family Hydrophilidae (Coleoptera) in Egypt. Ph.D. Thesis, Ain Shams University, Cairo, Egypt.

SATô M. 1972. The georissid beetles of Japan. Journal of Nagoya Women's College 18: 207-213. 
SCHALLER J.G. 1783. Neue Insekten. Abhandlungen der Hallischen Naturforschenden Gesellschaft 1: $217-332$.

SCHIÖDTE J.M.C. 1866. De tunnelgravende biller Bledius, Heterocerus, Dyschirius, og deres danske arter. Naturhistoriska Tidsskrift 4(3): 141-167.

SEMEnov A.P. 1900. Coleoptera asiatica nova. X. Horae Societatis Entomologicae Rossicae 34: $577-605$.

SHARP D.S. 1903. Water-beetles (Dytiscidae \& Hydrophilidae) of the Swedish Zoological expedition to Egypt and the White Nile. [in:] L.A. JÄGERSKIÖLD (ed.). Results of the Swedish Zoological expedition to Egypt and the White Nile 1901 under the direction of L.A. JAGERSKIOLD. Part I. Library of the Royal University of Uppsala, Uppsala, 1-10.

SHARP D.S. 1915. Studies in Helophorini. 5. The Trichelophori. The Entomologist's Monthly Magazine 51: 156-163.

SHARP D.S. 1916. Studies in Helophorini. 10. Helophorus (continued). The Entomologist's Monthly Magazine 52: 164-177.

SKALICKÝ S. 1999. New species of Heteroceridae from Thailand and Namibia (Coleoptera: Heteroceridae). Koleopterologische Rundschau 69: 119-123.

SKALICKÝ S. 2001. Faunistic notes on African Heteroceridae with description of Heterocerus ottomerkli sp. n. and redescription of $H$. dayremi PEYERIMHOFF (Coleoptera: Heteroceridae). Koleopterologische Rundschau 71: 143-148.

SkalickÝ S., Chikatunov V., PavlíčEK T. 2002. New records of Heteroceridae (Coleoptera) from Israel. Zoology in the Middle East 27(1): 117-118.

Spangler P.J., Staines C.L., Spangler P.M., Staines S.L. 2001. A checklist of the Limnichidae and the Lutrochidae (Coleoptera) of the world. Insecta Mundi 15: 151-165.

STEPHENS J.F. 1828. Illustrations of British Entomology; or, a synopsis of indigenous insects containing their generic and specific distinctions; with an account of their metamorphoses, times of appearance, localities, food, and economy, as far as practicable. Mandibulata. Vol. 2. Baldwin and Cradock, London.

StOREY G. 1916. List of Egyptian insects in the collection of the Ministry of agriculture. Government Press. no. 5. Technical and Scientific Service, Entomological Section, Cairo.

TAŞAR G.E. 2014. A contribution to the knowledge of Turkish Dryopidae, Elmidae and Heteroceridae (Coleoptera: Byrrhoidea) fauna. Archives of Biological Science Belgrade 66 (4): 1473-1478.

Thomson C.G. 1853. Review of those in Sweden found species of the family Palpicornia. Öfversigt af Kongliga Vetenskaps-Akademiens Förhandlingar 10: 40-58. [in Swedish]

Thomson C.G. 1859. Skandinaviens Coleoptera, synoptiskt bearbetade. Tom I. Berlingska Boktryckeriet, Lund.

THUNBERG C.P. 1784. Novae insectorum species descriptae. Nova Acta Regiae Societatis Scientiarum Upsaliensis 4: 1-28.

TOuRnIER H. 1868. Description des Dascillides du Bassin du Leman. Association Zoologique du Léman. Georg and F. Savy, Bâle - Genève - Paris.

Vondel B.J. VAN 2005. Water beetles from Bénin (Coleoptera: Haliplidae, Dytiscidae, Noteridae, Hydraenidae, Hydrochidae, Hydrophilidae, Gyrinidae, Elmidae). Deinsea 11: 119-138. 
WALTL J. 1838. Verzeichniss der um Passau vorkommenden seltenen Käfer nebst Beschreibung der neuen Arten. Isis von Oken 4(31): 263-273.

WinkLer A. 1932. Catalogus Coleopterorum, Regionis Palaearticae. A. Winkler, Vienna.

Yano K., Chu Y.I., SAto M. 1983. Faunal and biological studies on the insects of paddy fields in Asia. XI. Records on aquatic Coleoptera from paddy water in the world. Chinese Journal of Entomology 3(1): 15-31.

Zaitzev F.A. 1908. Catalogue des Coléopteres aquatiques des familles des Dryopidae, Georyssidae, Cyathoceridae, Heteroceridae et Hydrophilidae. Horae Societatis Entomologicae Rossicae 38: 283-420.

Zaitzev F.A. 1910. Coleopterorum Catalogus. Pars 17. Dryopidae, Cyathoceridae, Georyssidae, Heteroceridae. W. Junk, Berlin.

Zalat S., Gilbert F., Fadel H., El-Hawagry M.S., Saleh M., Kamel S., Gilbert J. 2008. Biological explorations of Sinai: flora and fauna of Wadi Isla and Hebran, St. Katherine Protectorate, Egypt. Egyptian Journal of Natural History 5(1): 6-15.

ZinetTi F., Terzani F. 2009. Coleotterofauna di due Riserve Naturali della Provincia di Arezzo (Toscana) con particolare riguardo alle loro zone umide (Insecta, Coleoptera). Quaderni Della Stazione Di Ecologia Del Civico Museo Di Storia Naturale Di Ferrara 19: 5-48.

Received: 24 February 2017

Accepted: 24 March 2017 